\title{
Encuesta seroepidemiológica de pian en la Costa Pacífica colombiana
}

\author{
Marcos Restrepo ${ }^{4}$, Berta N. Restrepo ${ }^{1}$, Beatriz Orozco 2, Julio C. Padilla ${ }^{3}$, \\ Claudia M. Cuervo ${ }^{1}$, Gilberto Alvarez ${ }^{3}$ \\ 1 Instituto Colombiano de Medicina Tropical, Medellín, Colombia. \\ ${ }^{2}$ Laboratorio de Salud Pública de Antioquia, Dirección Seccional de Salud de Antioquia, Medellín, Colombia. \\ ${ }^{3}$ Ministerio de Salud, Bogotá, D.C., Colombia. \\ El pian ha sido endémico en la Costa Pacífica colombiana, pero actualmente su frecuencia real \\ es desconocida, razón por la cual se realizó un estudio en las comunidades de esta zona del \\ país donde se habian conocido casos de pian. Se hizo búsqueda activa de casos clínicos \\ sospechosos a través de examen físico de personas con problemas dermatológicos; a todos \\ ellos se les practicaron exámenes serológicos de VDRL y FTA-ABS. Por cada caso clínico \\ sospechoso de pian, se estudiaron - por clínica y serología - 4 contactos intradomiciliarios y 42 \\ contactos extradomiciliarios. De las 1.830 personas examinadas, sólo 6 fueron reactivas a las \\ pruebas de VDRL y FTA-ABS, lo cual representa una prevalencia de treponematosis de $0,3 \%$. \\ Ninguna de las pruebas serológicas fue reactiva en los casos clínicos sospechosos de pian. \\ Esto nos lleva a concluir que, en la Costa Pacífica colombiana, el pian no es un problema de \\ salud pública y que se registran como pian otras enfermedades cuando el diagnóstico tiene sólo \\ bases clínicas.
}

Palabras clave: treponematosis, pian, seroepidemiología.

\begin{abstract}
Yaws seroepidemiological survey in the Pacific Coast of Colombia
Yaws has been endemic in the Pacific Coast of Colombia, but the actual prevalence is not known. For this reason we carried out a survey in the communities of this part of the country where cases had been observed. The active search for cases was based on clinical observations of patients with dermatological disorders, to whom VDRL and FTA-ABS tests were administered. The epidemiological survey included 4 intradomiciliary cases for each of the clinical patients detected and also 42 extradomiciliary cases. From a total of 1,830 persons examined, only 6 presented positive results for the VDRL and FTA-ABS tests, which represents a treponema infection prevalence of $0.3 \%$. None of the clinically suspected yaw cases reacted positively to the mentioned tests. These findings permit us to conclude that yaws is not a public health problem in the Pacific Coast of Colombia, and that the sole use of clinical parameters induces to mistaken diagnosis.
\end{abstract}

Key words: treponematosis, yaws, seroepidemiology.

El pian es una treponematosis endémica que se presenta en áreas tropicales húmedas, coincidiendo con asentamientos de poblaciones marginadas y con pocos servicios de salud de algunos países de Africa, Suramérica, el Caribe, el sudoeste de Asia y Oceanía (1,2).

\footnotetext{
$\overline{\text { Correspondencia: }}$

Marcos Restrepo, apartado aéreo 52162, Medellín, Colombia; fax: (574) 3014300.

icmt@epm.net.co
}

Recibido: 05/02/01; aceptado:29/05/01
La enfermedad también se conoce con los nombres de bubas o frambesia tropical. Es causada por la espiroqueta Treponema pallidum, subespecie pertenue, morfológicamente indiferenciable de la otra subespecie de T.pallidum que es Treponema pallidum endemicum; además, está Treponema carateum (3). Su transmisión se hace por cualquier contacto directo, no necesariamente de tipo venéreo, a través de las lesiones cutáneas o sus exudados por donde salen las espiroquetas. El contacto se favorece 
por hacinamiento o agua de baño contaminada con material de las lesiones, como puede ocurrir entre niños pequeños. Se ha observado que la transmisión es más frecuente en las épocas de lluvia (4).

Después de 2 a 5 semanas de incubación aparecen las primeras lesiones que constituyen el período primario de la enfermedad. Se observa inicialmente una pápula vegetante, llamada buba madre o pianoma primario, que exuda un líquido amarillento, maloliente y contiene abundantes treponemas, pero las lesiones pueden desaparecer espontáneamente en los primeros 6 meses $(4,5)$.

Varias semanas o meses después, se presenta el período secundario que se caracteriza por nuevas lesiones alrededor de la buba madre, denominadas bubas hijas o pianomas secundarios, que se diseminan por todo el cuerpo y se describen como máculas y pápulas descamativas, generalmente dolorosas. Además, puede existir fiebre, adenopatías, dolor óseo nocturno y periostitis (5).

El estado terciario se presenta después de 5 años y se caracteriza por la formación de gomas cutáneos y óseos, principalmente en las piernas. Hay hiperqueratosis de palmas y plantas, periostitis, osteítis, nodulaciones fibromatosas en articulaciones y, en algunos casos, hidrartrosis. Cuando las lesiones destructivas afectan la cara, hay daño y deformación de la nariz, los maxilares, el paladar y la faringe, padecimiento que se describe como la forma 'gangosa' del pian. No existe compromiso visceral ni manifestaciones neurológicas $(4,5)$. El tratamiento de elección ha sido la penicilina benzatínica que se continúa utilizando aún para los tratamientos masivos, base del control del pian en las zonas endémicas $(2,5)$.

El diagnóstico es principalmente clínico, ya que no se dispone de un método de laboratorio de gran especificidad. Dependiendo del estado de evolución, se debe diferenciar de pioderma, úlcera tropical, úlcera de la anemia de células falciformes, leishmaniosis cutánea, micosis, lepra, tuberculosis cutánea y sífilis venérea. La histopatologia es semejante a la de la sífilis. Se encuentra hiperplasia epitelial en las lesiones con infiltrado de células mononucleadas, en tanto que la endarteritis sólo se presenta en las formas tardías (5). Los estudios microbiológicos, como el examen directo al campo oscuro, que confirma la presencia de treponemas, es difícil de realizar en condiciones de campo, pero los métodos serológicos para sífilis tienen valor para complementar el diagnóstico de género mas no de especie; las pruebas no treponémicas (VDRL) tienen una sensibilidad entre el 70 y el $99 \%$, y las treponémicas (FTA-ABS) entre el 85 y el $100 \%$ $(2,5)$.

El pian es endémico en zonas selváticas de algunos países tropicales de Africa, Asia y América, con temperaturas elevadas, alta precipitación pluvial y, por tanto, humedad. Los tratamientos masivos con penicilina reducen rápidamente la enfermedad en las comunidades. En el período de 1950 a 1970, se promovieron campañas de control de las treponematosis endémicas o no venéreas con penicilina y se observó una rápida disminución en la prevalencia de infecciones activas y latentes (2). En zonas hiperendémicas de Nigeria septentrional, pasaron de una prevalencia de 4,2 a $0,02 \%$; en Samoa occidental, de 3,0 a $0,005 \%$, y, en Filipinas, de 0,1 a $0,01 \%(2,6)$. En el hemisferio occidental, con tratamientos en masa, la incidencia, que era de más de 44.000 casos por año en el período 1950-1954, bajó a 437 casos en 1975 (7). En 1982, la incidencia notificada de pian en las Américas fue muy baja, con pequeños focos en Brasil, Colombia, Ecuador, Guyana, Surinam, Haití y en las islas de Dominica y Martinica (6).

En Colombia, el pian ha sido endémico a lo largo de la Costa Pacífica y existen descripciones clínicas entre los años 1801 y 1889. Alrededor de 1930, se iniciaron en el país las campañas de tratamiento antipiánico con arsénico y bismuto (8) y, en 1936, se estimó que existían 70.000 casos de pian en los departamentos de Valle, Cauca, Nariño y Chocó, la mayoría en estos últimos dos departamentos $(9,10)$. En 1954, se informaron 2.473 casos, 225 en 1964 y bajaron a 22 en 1974 (9). El territorio en donde se encuentra la infección por pian corresponde a zonas húmedas, cálidas y con selva tropical, habitadas principalmente por población de raza negra y comunidades indígenas. 
El Ministerio de Salud de Colombia estableció una campaña antipiánica en 1950 y, en 1963, la transfirió como programa al Servicio de Erradicación de la Malaria (SEM). El control de la enfermedad se ha basado en el tratamiento con penicilina benzatínica de los casos clínicos detectados por promotores de saneamiento, administrando el medicamento en dosis única por vía parenteral, pero no se realiza estudio microbiológico ni serológico $(11,12)$.

Desde la iniciación de la campaña, el pian ha mostrado una disminución de su incidencia general; sin embargo, a partir de la década del 70 la disminución de los casos nuevos se atribuyó al subregistro por deterioro de las actividades de búsqueda, lo cual ha hecho que no se conozca la frecuencia de la enfermedad. En los últimos 10 años, se informaron 724 casos, siendo el año de 1989 el de mayor incidencia con 1,3/100.000 hab (407 casos). En 1992, se registraron 108 casos, con una tasa de 0,3/100.000 hab (11).

El objetivo del presente estudio es definir el estado actual de las treponematosis y, especialmente, del pian en la Costa Pacífica colombiana. Se plantea la búsqueda activa de casos, aprovechando las actividades de control de otras enfermedades y una encuesta seroepidemiológica a los contactos intradomiciliarios y extradomiciliarios a partir de los casos clínicamente sospechosos. Se considera que no existe treponematosis si las pruebas serológicas no son reactivas.

\section{Materiales y métodos}

Se realizó un estudio de tipo descriptivo y de corte. Los funcionarios de Campañas Directas del Ministerio de Salud de cada departamento visitaron las comunidades de la Costa Pacífica colombiana en donde se habían informado casos de pian en 1992 y 1993. En estas comunidades se hizo búsqueda de pacientes que tuvieran lesiones dermatológicas, clínicamente sospechosas de pian. Se consideró como caso clínico sospechoso de pian al paciente que presentara lesiones compatibles: bubas madres, bubas hijas, gomas cutáneos, úlceras, clavos, lesiones destructivas en la cara o lesiones condilomatosas exudativas en los orificios naturales. Los funcionarios de las zonas endémicas fueron instruidos por la médica dermatóloga para la búsqueda de personas con lesiones por esta enfermedad. En la encuesta, también se consignó el dato sobre la percepción que la población tenía sobre el antecedente de haber sufrido pian, leishmaniosis o sífilis.

A cada paciente se le tomó una muestra de sangre para estudio serológico de VDRL y FTA-ABS. Por cada caso clínico sospechoso de pian, se estudiaron 5 contactos intradomiciliarios y 50 contactos extradomiciliarios de la vecindad. A todos se les realizó una encuesta sobre aspectos demográficos, clínicos y epidemiológicos. Se excluyeron las personas sin lesiones y sin historia clínica de haber padecido pian.

Contacto intradomiciliario era la persona, familiar o no, que hubiera compartido el mismo techo con el caso clínico sospechoso de pian durante un mínimo de tres meses. Contacto extradomiciliario era la persona, familiar o no, que no convivía bajo el mismo techo con el caso clínico sospechoso, pero tenía relación social, institucional o geográfica con el caso clínico.

El caso positivo para treponematosis, se refiere a la persona con VDRL y FTA-ABS reactivos. El caso positivo para pian debía tener lesiones dermatológicas compatibles con la enfermedad y las pruebas de VDRL y FTA-ABS reactivas $(4,5)$. A los pacientes con lesiones no se les realizó estudio de campo oscuro para búsqueda de espiroquetas por la carencia de equipo adaptable a las condiciones de la zona selvática en donde habitan las comunidades.

La muestra de sangre para el estudio serológico se obtuvo por punción capilar del dedo índice, impregnando cuatro círculos de papel de filtro (Whatman No. $3 \mathrm{MM} \mathrm{CHr}$ ) con un diámetro de 1,5 $\mathrm{cm}$ de diámetro cada uno (13-15). Cada círculo absorbe aproximadamente $50 \mu$ l de sangre, lo cual corresponde a alrededor de $62 \%$ de suero, calculando que la persona de la zona tiene un hematocrito promedio de $38 \%$. Una vez secó la sangre a temperatura ambiente, las muestras se almacenaron a $4^{\circ} \mathrm{C}$ en bolsas plásticas y se transportaron en frío al Instituto Colombiano de 
Medicina Tropical en Medellín, en donde se congelaron a $-20^{\circ} \mathrm{C}$ hasta su procesamiento.

Para realizar las pruebas, la sangre fue eluida del círculo de papel agregando $248 \mu$ l de solución tampón de fosfatos (PBS) con Tween $80 \%$, pH 7,2 , durante 24 horas a $4^{\circ} \mathrm{C}$, con lo cual se obtuvo una dilución de 1:8.

Las pruebas serológicas para la detección de anticuerpos anti-treponema fueron el VDRL y el FTA-ABS. La prueba de VDRL se realizó con antígeno cardiolipínico preparado por el Instituto Nacional de Salud de Colombia. Se utilizó la técnica de floculación en placa recomendado por el Laboratorio de Enfermedades Venéreas del Servicio de Salud Pública, EE.UU. (16), con previa inactivación a $56{ }^{\circ} \mathrm{C}$ durante 30 minutos. Cada una de las muestras se diluyó a la mitad para realizar la cuantificación de anticuerpos en términos de dilución. Las muestras reactivas se procesaron por el método del FTA-ABS, con eluidos que tuvieran la dilución 1:5 siguiendo la técnica recomendada por los Centros Internacionales de Enfermedades de Transmisión Sexual $(16,17)$ y que se ha utilizado para hacer esta reacción en muestras recolectadas en papel de filtro $(14,15)$. El antígeno utilizado fue T. pallidum, cepa Nichols, fijados en placa (Trepo-spot IF, Laboratoires Biomérieux ${ }^{\circledR}$, Francia), sorbente y conjugado antigammaglobulina humana (Fluoline$G$, Biomérieux $®$, Francia). La lectura se hizo en microscopio de fluorescencia (Nikon $\AA$, Japón), con objetivo UV 40X, bloque de filtros DM 510. Como controles positivo y negativo, tanto de las pruebas treponémicas como las no treponémicas, se utilizaron sueros de pacientes conocidos con las pruebas de VDRL y FTA-ABS reactivas y no reactivas.

\section{Resultados}

La encuesta se realizó en 1995 en la Costa Pacífica colombiana. En total, se estudiaron 1.830 personas; $981(53,8 \%)$ estaban ubicadas en 27 localidades de 8 municipios del departamento de Chocó; 725 (39,6\%), en 14 localidades de 3 municipios del departamento de Nariño, y 124 $(6,8 \%)$, en 2 localidades de un municipio del departamento de Cauca. Se estudiaron 42 localidades en 12 municipios.
Los grupos de edad aparecen en la figura 1, en donde se observa que la mayoría de la población estuvo representada por los grupos de 5 a 44 años. El promedio de edad fue de 24,23 , con una edad mínima de 9 meses y una máxima representada por una mujer de 102 años. El $50 \%$ de la población tenía más de 17 años (mediana=17) y la edad más frecuente fue la de 12 años (moda=12). En cuanto a sexo, la participación de la mujer fue mayor con $56,2 \%$.

La mayoría de personas en Chocó y Cauca residian en la zona rural municipal, 78,0 y $99,2 \%$, respectivamente; por el contrario, en Nariño el $98,3 \%$ eran de la zona urbana. La distribución por oficio habitual mostró que la ocupación de estudiante predominó con un $28,7 \%$, seguidas en frecuencia por las amas de casa con $24,6 \%$; luego, los agricultores, $18,6 \%$, y los mineros, $7,7 \%$. Los maestros de escuela fueron pocos, $1,1 \%$; los comerciantes, $0,5 \%$, y los pescadores, $0,4 \%$. Las mujeres desempeñaron todos los oficios, menos la agricultura. Los hombres, en su mayoría agricultores y estudiantes, desempeñaban todos los oficios, incluso el de ama de casa.

En el cuadro 1 vemos que del total de personas estudiadas, 38 fueron clasificadas como casos clínicos sospechosos de pian cuando se hizo el examen físico. Se captaron 164 contactos intradomiciliarios y 1.611 contactos extradomiciliarios. El promedio de contactos intradomiciliarios por caso sospechoso fue de 4 y el de contactos extradomiciliarios fue de 42 .

Las lesiones semejantes a pian descritas por los encuestadores en los 38 casos sospechosos

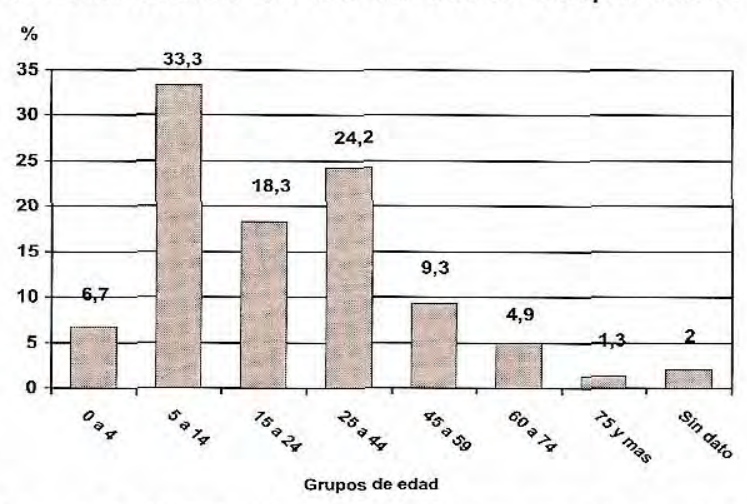

Figura 1. Distribución porcentual por grupos de edad. $\mathrm{n}=1.830$ 
Cuadro 1. Distribución de los encuestados por departamentos de la Costa Pacífica.

\begin{tabular}{|c|c|c|c|c|c|c|c|c|}
\hline \multirow[t]{3}{*}{ Departamento } & \multicolumn{8}{|c|}{ Clasificación de encuestados } \\
\hline & \multicolumn{2}{|c|}{ Caso clínico } & \multicolumn{2}{|c|}{$\begin{array}{c}\text { Contactos } \\
\text { intradomiciliarios }\end{array}$} & \multicolumn{2}{|c|}{$\begin{array}{c}\text { Contactos } \\
\text { extradomiciliarios }\end{array}$} & \multicolumn{2}{|c|}{ Sin dato } \\
\hline & $\mathbf{n}$ & $\%$ & $\mathbf{n}$ & $\%$ & $\mathbf{n}$ & $\%$ & $\mathbf{n}$ & $\%$ \\
\hline Nariño & 10 & 26,3 & 40 & 24,4 & 673 & 41,8 & 2 & 11,8 \\
\hline Cauca & 3 & 7,9 & 15 & 9,1 & 105 & 6,5 & 1 & 5,9 \\
\hline Chocó & 25 & 65,8 & 109 & 66,5 & 833 & 51,7 & 14 & 82,4 \\
\hline Total & 38 & 100,0 & 164 & 100,0 & 1611 & 100,0 & 17 & 100,1 \\
\hline
\end{tabular}

$\mathrm{n}=1.830$

fueron, en orden de frecuencia: gomas, $31,6 \%$; úlceras, $28,9 \%$; bubas secundarias, $15,8 \%$; clavos, $13,2 \%$; otras lesiones, $13,2 \%$, y buba madre, 2,6\%. La localización anatómica de estas lesiones correspondió a los miembros superiores, miembros inferiores, cuello y cara.

Otro aspecto estudiado fue la percepción que la población tenía sobre el antecedente de haber sufrido pian, leishmaniosis o sífilis; se encontró que el $8,9 \%$ dijo haber sufrido pian. El 7,4\% afirmó que tuvo leishmaniosis y el $0,8 \%$, sífilis.

Al realizar el examen del VDRL, se detectaron 39 personas reactivas en la población estudiada en la Costa Pacífica, que corresponde al 2,1\% de todas las personas estudiadas; en este grupo, el título de la reacción fue de 1:8, con excepción de tres personas que tuvieron títulos mayores de hasta 1:256. La distribución de la reactividad a la prueba de VDRL por departamentos muestra que en Nariño y Cauca fue baja, 0,3 y $0,8 \%$, respectivamente, pero de 3,7\% en Chocó.

A las 39 personas reactivas al VDRL se les realizó la prueba de FTA-ABS para comprobar treponematosis, en la cual se incluye sífilis y pian; solamente se encontraron 6 personas reactivas a la inmunofluorescencia. Esto representó una prevalencia de treponematosis del $0,3 \%$ para el total de la población estudiada. Al discriminar por departamentos, la prevalencia para Chocó fue de 0,4\% (4 personas); para Cauca, $0,8 \%$ (1 persona), y para Nariño, $0,1 \%$ ( 1 persona).

En el análisis de los municipios donde se hallaron los casos positivos, se encontró que la mayor prevalencia de positividad para treponematosis fue en Lloró, localidad de Ogadó (Chocó) con 2,8\%, seguido por Bajo Baudó, localidad de Bazarudo de Barró (Chocó) con 1,0\%; Quibdó, localidad de Chiguarandó (Chocó) y Timbiquí, localidad de Pizare (Cauca) con 0,8\%, y Santa Bárbara, localidad de La Piedra con $0,2 \%$. Ninguno de los casos con las pruebas serológicas reactivas mostraron lesiones cutáneas y, por tanto, se descartaron lesiones activas de pian o secuelas antiguas de esta enfermedad. Aquellos 38 pacientes en quienes se describieron lesiones clínicamente compatibles con pian, no presentaron serologías treponémica y no treponémica reactivas; por tanto, no tenían en ese momento, ni habían padecido anteriormente, una treponematosis.

De los contactos intradomiciliarios con pacientes sospechosos, 4 fueron reactivos al VDRL pero sólo 1 de ellos presentó la reacción de FTA-ABS reactiva, lo cual muestra que los contactos no tuvieron relación con transmisión de pian (cuadro 2). De los contactos extradomiciliarios,

Cuadro 2. Distribución de la reactividad con VDRL y FTA-ABS en todos los grupos por departamento.

\begin{tabular}{|c|c|c|c|c|c|c|c|c|}
\hline \multirow[t]{2}{*}{ Departamento } & \multicolumn{2}{|c|}{$\begin{array}{c}\text { Casos clínicos } \\
\text { sospechosos }\end{array}$} & \multicolumn{2}{|c|}{$\begin{array}{l}\text { Contactos } \\
\text { intradomiciliarios }\end{array}$} & \multicolumn{2}{|c|}{$\begin{array}{c}\text { Contactos } \\
\text { extradomiciliarios }\end{array}$} & \multicolumn{2}{|c|}{ Total } \\
\hline & VDRL & FTA & VDRL & FTA & VDRL & FTA & VDRL & FTA \\
\hline Nariño & 0 & 0 & 0 & 0 & 2 & 1 & 2 & 1 \\
\hline Cauca & 0 & 0 & 0 & 0 & 1 & 1 & 1 & 1 \\
\hline Chocó & 0 & 0 & 4 & 1 & 32 & 3 & 36 & 4 \\
\hline
\end{tabular}


35 fueron reactivos al VDRL y, de ellos, 5 lo fueron también al FTA-ABS, lo cual confirma que la prevalencia real de treponematosis fue de $0,3 \%$, posiblemente por sífilis. Estos individuos con serología reactiva, correspondieron a 3 menores de 10 años y a otros 3 mayores de 50 años, ninguno con lesiones en piel; solamente un adulto apareció con cicatriz y antecedente de haber sufrido pian hace muchos años, por lo cual fue clasificado como secuela de pian.

\section{Discusión}

El pian ha sido endémico en toda la Costa Pacífica desde el departamento de Nariño en límites con Ecuador, provincia de Esmeraldas, hasta Chocó en sus límites con Panamá. En la zona del sur del país se han informado más casos que en las otras zonas $(7,11,12)$. En Ecuador, provincia de Esmeraldas, limítrofe con Colombia, se estudió en 1991 el foco más recientemente localizado. Los habitantes pertenecían a comunidades negras de la hoya del río Santiago, en donde se encontró una seropositividad del $94,9 \%$ con una prevalencia de lesiones activas de pian del $11,3 \%$. El río Santiago se había considerado una región hiper-endémica para pian con prevalencia de 17,9 y seropositividad de $98,0 \%$. La mayor incidencia estuvo en el grupo de niños entre 5 y 12 años con un 55,8\% de lesiones clínicas, la mayoría de ellas localizadas en las piernas. Los papilomas fueron las lesiones más prevalentes con $55,4 \%$ (18).

Las manifestaciones clínicas de pian se han considerado lo suficientemente claras para distinguirlas de las lesiones de otras enfermedades, cuando se estudian las comunidades en zonas endémicas con personal capacitado para este diagnóstico. No obstante lo anterior, cuando la infección baja en frecuencia y el personal que identifica las lesiones cambia, se presenta la dificultad de conseguir nuevo personal con la posibilidad de hacer diagnóstico clínico veraz, lo que hace que el uso de pruebas de laboratorio sea indispensable para confirmar los casos de esta treponematosis (15). El diagnóstico por medio de microscopio de campo oscuro para observar el microorganismo confirmaría la presencia de la espiroqueta del género Treponema en material exudativo de las lesiones (15); sin embargo, este procedimiento es difícil de llevar a cabo en las zonas selváticas y rurales en donde se encuentran los casos. Como alternativa al examen microbiológico, se dispone de la detección de anticuerpos para los microorganismos del género, técnica de las pruebas de VDRL como indicación presuntiva, con una buena sensibilidad para las treponematosis (4) y la reacción de FTA-ABS como prueba confirmatoria de una alta especificidad para las treponematosis, aunque no se puede separar la infección de las diferentes especies patógenas de Treponema (4).

Como las pruebas utilizadas tienen un alto grado de sensibilidad y especificidad, la ausencia de anticuerpos anti-Treponema indicaría que no existe una infección treponémica, como lo serían el pian, la sífilis o el carate. La presencia de anticuerpos habla de infección y las características clínicas y epidemiológicas ayudan a precisar las lesiones de pian. Con el presente estudio, se define que la frecuencia de las treponematosis ha bajado en la Costa Pacífica colombiana a través de los años y la prevalencia encontrada en nuestro trabajo es de $0,3 \%$.

Se recomienda no utilizar la sola observación clínica para informar los casos de pian, pues se puede estar falseando la información por falta de experiencia clínica del personal auxiliar que busca a los enfermos dada la ausencia de casos activos de pian, confundiéndolo con otras lesiones que no corresponden a esta enfermedad. Por tanto, es importante utilizar la ayuda del laboratorio para sustentar el registro de los casos, pues, como lo demostró nuestro trabajo, es un error frecuente que los encargados del programa registren como pian afecciones que, una vez realizado el estudio serológico, demuestran no corresponder a lesiones de una treponematosis. En igual forma, se interpreta la presencia de una serología reactiva sin lesiones activas, lo cual corresponde a secuelas antiguas de pian u otra treponematosis.

Se considera que, actualmente, el pian no es un problema de salud pública en la Costa Pacífica colombiana y que para vigilar el control y posible erradicación de la enfermedad, se debe estudiar el caso sospechoso con pruebas serológicas 
como el VDRL, que tiene una alta sensibilidad, y con una prueba confirmatoria como FTA-ABS, que define una treponematosis $(2,5)$; de lo contrario, se continuarán haciendo registros de casos falsos positivos y no se certificará la incidencia real. Esta vigilancia se debe hacer con un procedimiento fácil, de bajo costo y eficiente, como es la toma de muestra de sangre total con papel de filtro, que cualquier auxiliar de campo puede tomar y que es posible remitir por correo a un centro donde se realice la serología $(14,15)$. Este sistema de vigilancia se debe integrar a la atención primaria de cada localidad, como lo han hecho algunos investigadores en las islas Solomon (19); además, se debe acompañar de la administración de tratamiento con penicilina benzatínica $(4,5,18-20)$. En la provincia de Esmeraldas, Ecuador, se disminuyó la prevalencia de lesiones cutáneas de 11,2 a $0,6 \%$ en un período de 5 años utilizando tratamientos masivos (21).

\section{Agradecimientos}

A la Organización Panamericana de la Salud (OPS) y al Ministerio de Salud de Colombia por la financiación del estudio y por la asesoría, especialmente a Agustín Lago; a los Servicios Seccionales de Salud de los departamentos de Cauca, Valle, Chocó y Nariño, cuyos funcionarios de Campañas Directas, del SEM y del Programa de Malaria coloboraron en la recolección de la información.

\section{Referencias}

1. Hopkins DR. After smallpox eradication: Yaws? Am J Trop Med Hyg 1976;25:860.

2. OMS. Treponematosis. Informe de un Grupo Científico de la Organización Mundial de la Salud. Serie Informes Técnicos No. 674. Ginebra: OMS; 1982.

3. Holt JG, Krieg NR, Sneath PHA, Staley JT, Williams ST. Bergey's manual of determinative bacteriology. Ninth edition. Baltimore: Williams \& Wilkins; 1994. p.30-7.

4. Perine PL, Hopkins DR, Niemel PLA, St. John RK, Causse G, Antal GM. Manuel des tréponématoses endémiques: pian, syphilis endémique et pinta. Geneva: Organization Mondiale de la Santé; 1985.

5. Chulay JD. Treponema species (yaws, pinta, bejel). En: Mandel GL, Douglas RG, Bennett JE, editors. Principles and practice of infectious diseases. Third edition. New York: Churchill Livingtone; 1999. p.1329-30, 1809-10.
6. OMS. Investigaciones sobre las treponematosis. Informe de un Grupo Científico de la Organización Mundial de la Salud. Serie Informes Técnicos No. 455. Ginebra: OMS; 1970.

7. Hopkins DR. Yaws in the Americas, 1950-1975. J Infect Dis 1977;136:548-54.

8. Narváez GL. Pian y campaña antipiánica en Colombia. Rev Fac Med Bogotá 1956;24:294-342.

9. Hopkins DR, Flórez D. Pinta, yaws, and venereal syphilis in Colombia. Internat J Epidemiol 1977;6:349-55.

10. Torrijos RC. Pliego de conclusiones de la conferencia antipiánica reunida en Buenaventura en los días 26 y 27 de junio de 1936. Rev Higiene Bogotá 1936;17:111-27.

11. Ministerio de Salud. Salud en Colombia. Diez años de información (documento interno). Santa Fe de Bogotá: Ministerio de Salud; 1994.

12. Ministerio de Salud. Epidemiología y control del pian en Colombia (documento interno). Santa Fe de Bogotá: Ministerio de Salud; 1994.

13. Guimaraes MCS, Camargo ME, Ferreira AW, Ayres de Castillo E, Nakahara OS. Comparison of IgG and IgM contents in serum and filter paper blood eluates. Am J Trop Med Hyg 1978;27:350-3.

14. Hopkins DR. Fluorescent treponemal antibody absorption (FTA-ABS) tests using blood samples collected on filter paper. Am J Trop Med Hyg 1977;26: 188-9.

15. Perine PL, Nelson JW, Lewis JO, Liska S, Hunter EF, Larsen SA, et al. New technologies for use in the surveillance and control of yaws. Rev Infect Dis 1985;7: S295-9.

16. Public Health Service of the United States. Manual of test for syphilis. Publication No. 411. Washington, D.C.: Public Health Service; 1969.

17. Hunter EF, Deacon W, Meyere P. An improved FTA/fta test for syphilis, the absortion procedure (FTA-ABS). Pub Hith Rep 1964;79:410.

18. Guderian RH, Guzmán JR, Calvopiña M, Cooper P. Studies on a focus of yaws in the Santiago Basin, province of Esmeraldas, Ecuador. Trop Geogr Med 1991; 43:142-7.

19. Fegan D, Glennon M, Macbride-Stewart G, Moore T. Yaws in the Solomon Islands. J Trop Med Hyg 1990;93:527.

20. Relvich AL. Observations on the rate of resolution of yaws lesions after procaine benzyl penicillin treatment. Trans R Soc Trop Med Hyg 1959;53:75-7.

21. Anselmi M, Araújo E, Narváez A, Cooper PJ, Guderian RH. Yaws in Ecuador: impact of control measures on the disease in the Province of Esmeraldas. Genitourin Med 1995;71:343-6. 